\title{
A flexible computer-controlled perception laboratory
}

\author{
STEVEN E. POLTROCK and WAYNE G. CARR \\ University of Denver, Denver, Colorado 80208
}

\begin{abstract}
Research in perception is often guided or limited by the capabilities of the laboratory. A computer-controlled perception laboratory is described that was designed to be extremely flexible at a modest cost. The laboratory permits control of onset time, offset time, and intensity of visual, auditory, and tactile stimuli. The visual stimuli include single points, text, stereoscopic displays, and two-dimensional representations of three-dimensional objects moving in space. The design of the hardware and software and the criteria that guided our choice of this design are discussed. Finally, potential applications of this laboratory are considered.
\end{abstract}

The expense of establishing a perception laboratory is prohibitive, encouraging either research involving minimum equipment or specialization in an affordable methodology. The capabilities of a perception laboratory tend to guide or limit the nature of research, discouraging or encouraging new avenues of investigation. This article describes our efforts to develop an extremely flexible, computer-controlled perception laboratory on a modest budget.

\section{LABORATORY CAPABILITIES}

The versatility of a computer-controlled laboratory may be restricted by limitations in any of three areas: (1) the stimulus alternatives or control of stimulus parameters, (2) the response alternatives, and (3) the software controlling the laboratory. The design of our laboratory is intended to achieve flexibility in each of these areas, with particular attention to the stimuli. Control of stimulus parameters is critically important in perceptual research. Much of our research is psychophysical, requiring control of the intensities, onset times, and offset times of simple visual, auditory, and tactile stimuli. To accommodate our other research interests, we require visual stimuli that include single points, text, and simple graphics. Furthermore, each kind of visual stimulus must be capable of movement and/or stereoscopic display. Our requirements for auditory stimuli are equally challenging. Usually, a simple sine wave or square wave with controlled intensity, onset time, and offset time is sufficient. However, some applications require more complex waveforms, such as speech or computer-generated functions. The tactile stimuli are simple in comparison. Vibrations with con-

We thank Dale Steffen of the Denver Research Institute, Robert Jackson, and Rick Obert for their help in developing this laboratory. The laboratory development was supported by the National Institute of Mental Health (Grant MH32037-01) and by a Biomedical Research Support grant from the National Institute of Health. trolled intensity, onset time, and offset time are sufficient.

We considered flexibility in the response alternatives to be less important. Currently, subjects are provided three binary response alternatives, and expansion to five alternatives is possible. Actually, five binary responses permit 31 response alternatives by coding a response as a combination of binary responses. The hardware to perform this conversion is not commercially available, but it is easily constructed. This conversion permits keyboard responses such as words or sentences. How ever, most perceptual research does not require verbal responses. The five binary responses provide sufficient alternatives to construct a rating scale without additional hardware.

The design of the software is similarly motivated by a need for flexibility. Typically, the choice of software represents a tradeoff between flexibility and simplicity for the user. We achieve flexibility by using FORTRAN to control the experiments. Thus, a FORTRAN program completely determines the sequence of events that comprise an experiment. To simplify the task of writing such a program, a library of assembly language routines is available to control the stimulus hardware, record responses, and time events. The design of this software is described in more detail later.

\section{HARDWARE}

With these ambitious requirements in mind, we considered how the necessary equipment could be acquired with a modest budget. Fortuitously, much of the equipment was available within the psychology department. An X-Y display with intensity control was found abandoned in the workshop, and a large sound-attenuating chamber was about to be dismantled to make room for some remodeling. The greatest saving was achieved by using the department's minicomputer, a PDP-11/10, to control the laboratory. In addition to these acquisitions, total equipment expenses were $\$ 3,000$, including 
$\$ 1,000$ to repair the X-Y display. Recognizing that few researchers are likely to be so fortunate, the sections below describe our equipment and the minimal equipment required to duplicate this system.

\section{Computer Hardware}

The PDP-11/10 minicomputer has $24 \mathrm{~K}$ of memory, two RK05 disk drives, a floppy drive, and a lab peripheral system that includes a programmable clock, digitalto-analog (D/A) and analog-to-digital (A/D) converters, and digital inputs and outputs. A four-channel D/A converter was the only addition to the computer.

The essential functions of the laboratory could be achieved with a DEC LSI-11 microprocessor with floppy disk drives, a programmable clock, an instrument interface, and four D/A channels. Presentation of digitized speech sounds or other complex waveforms requires a faster disk drive and an $A / D$ converter. The basic system can be purchased for less than $\$ 15,000$.

\section{Laboratory Hardware}

The laboratory hardware includes equipment to present visual, auditory, and tactile stimuli. Visual stimuli are presented on a Hewlett-Packard 1300A X-Y display. This device can illuminate any points of a 4,096 by 4,096 array on an $8 \times 10 \mathrm{in}$. screen, with independent control of the intensity of each point. The high point density provides the fine control of disparities required for stereoscopic displays. The $X$ and $Y$ coordinates of the display are controlled by two D/A converters. The intensity of the display may be controlled by a third converter, providing independent control of the intensity of each point. Alternatively, a fixed intensity level may be achieved, with a slight reduction in system overhead, by using the intensity channel provided with most $D / A$ converters.

Auditory stimuli are presented via a Telephonics TDH-39 headset. The auditory stimuli can be generated in two ways. Sounds can be output from the computer via a $\mathrm{D} / \mathrm{A}$ converter, permitting presentation of speech and other complex stimuli. Alternatively, the stimuli can be produced by one or more waveform generators. Computer-controlled solid state switches are used to select a waveform generator and to start and stop the stimulus.

The tactile stimuli are relatively simple. A Ling Model 203 shaker presents a vibratory stimulus to the skin. Just as for auditory stimuli, the shaker may be driven by either a $\mathrm{D} / \mathrm{A}$ converter or a waveform generator.

In each stimulus modality there are alternative methods for controlling stimuli. Choosing between these alternatives for an experiment requires consideration of two competing factors: (1) the degree of stimulus control required in the experiment, and (2) the amount of computer time required to achieve this control. The D/A converters permit control of the intensity of visual stimuli and also presentation of complex waveforms as auditory or tactile stimuli. Unfortunately, the computer does not have direct memory access, so operation of the converters requires computer time. Each time a device completes an input or output operation, the computer temporarily interrupts the normal flow of processing and executes a routine called a device handler. The time required by the device handlers and other system routines will be referred to as computer overhead. This computer overhead is a problem when auditory stimuli are presented because of the high data rate $(10 \mathrm{kHz})$ required to represent stimuli. Tactile stimuli are of lower frequency and can be represented with a sampling rate of $100 \mathrm{~Hz}$ or less, adding no substantial burden to the overhead. Fortunately, the increase in overhead to control visual intensity is also negligible. Our approach has been to use the D/A converters only when necessary. As a result, the software has not been developed to permit presentation of complex auditory stimuli and simultaneous presentation of stimuli in other modalities.

The mechanics of connecting signal sources to laboratory equipment are accomplished by means of the two patchboards shown in Figure 1. Coaxial cables run from the computer input and output devices to $\mathrm{BNC}$ connectors on the upper patchboard. Other coaxial cables

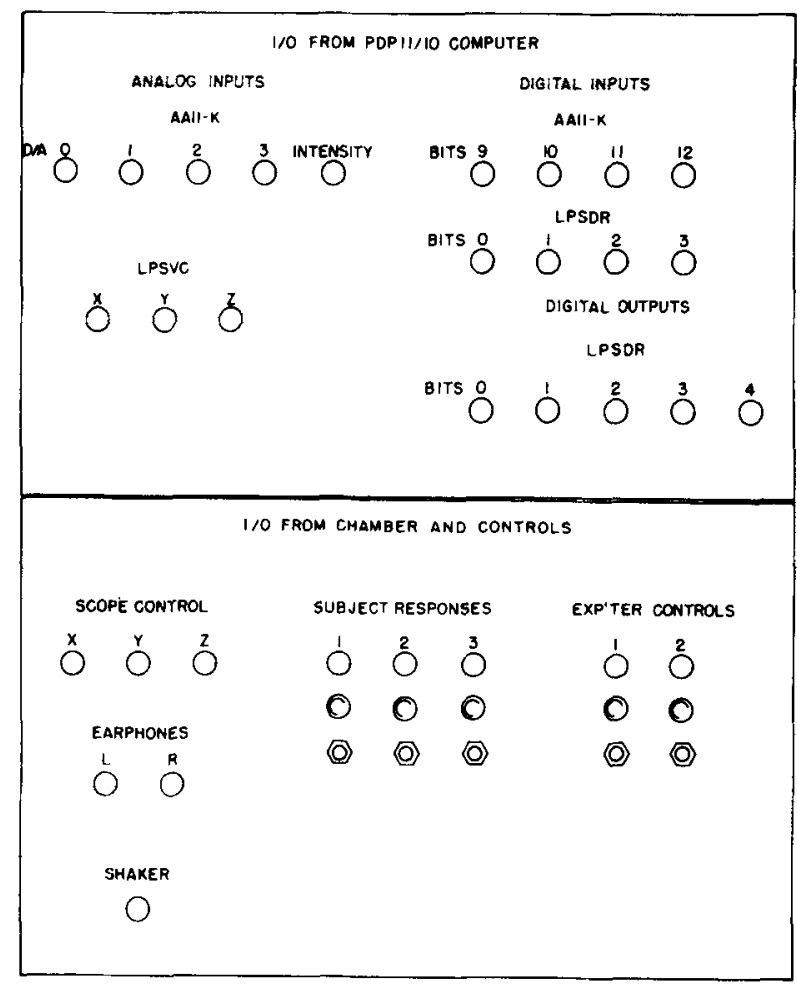

Figure 1. Patchboards are used to connect laboratory and computer devices. The upper board is connected to computer peripheral devices and the lower board is connected to the laboratory equipment. The circles represent BNC connectors used to connect a computer device to a laboratory device. The bottom two rows for subject responses and experimenter controls are indicator lights and microswitches. 
lead from connectors on the lower patchboard to the headset, the X-Y display, the shaker, and the response keys. The connections are established by means of jumper cables leading from one patchboard to the other. Similarly, other devices can be connected to the computer or the laboratory equipment by running cables to the patchboards. This arrangement permits changing a signal source in a matter of seconds without disturbing any of the cables connected to the laboratory or computer equipment. These patchboards were easily constructed from fiberboard.

\section{SOFTWARE}

Our PDP-11/10 uses the RT-11 Version 3 operating system, providing file management and some support for interrupt processing. All programs to conduct experiments are written in FORTRAN, providing the researcher considerable control over the sequence of events constituting the experiment. We have developed a library of routines intended to facilitate the programmer's task. Two kinds are available: (1) routines that interface with the laboratory hardware, and (2) general service routines for stimulus manipulations. Both kinds are described below.

\section{Laboratory Routines}

All computer input and output devices that interface with laboratory equipment are controlled by library routines written in macro assembly language. The description of these routines is brief because the functions provided by these routines and the software design are fairly commonplace in systems of this type. Further information is available upon request.

The library contains routines to initialize the system, measure time intervals, collect responses, and start or stop visual, auditory, and tactile stimuli. To display a visual stimulus, the user calls a routine and specifies vectors containing the $\mathrm{X}$ and $\mathrm{Y}$ display coordinates. The display is automatically refreshed until a routine is called that stops the display. An alternative display routine accepts a vector of intensity values in addition to the $\mathrm{X}$ and $\mathrm{Y}$ coordinates.

Auditory and tactile stimuli are presented by calling a routine and specifying a vector of values that constitute the waveform. For auditory stimuli the data rate is automatically set to $10 \mathrm{kHz}$, but for tactile stimuli the user specifies the data rate. These two routines are still under development.

The routines to time intervals and collect responses have alternative modes of operation. To wait a specified interval, the user calls a WAIT routine with the interval as an argument. Normally, the routine returns control to FORTRAN when the interval has elapsed. Alternatively, the user may specify a flag that is used to indicate the end of the interval. The WAIT routine sets the flag and immediately returns to FORTRAN. When the interval has elapsed the flag is cleared. Similar options exist for the RESPNS routine that collects responses. The user specifies locations for the latency and response and indicates which response keys will be monitored. The routine returns control when an acceptable response has occurred, or if a flag is specified, it returns immediately and clears the flag to indicate the response has occurred.

The design of the laboratory software is generally consistent with DEC's guidelines for routines that control input and output operations. The routines called by the experimenter's FORTRAN program simply initiate the input or output and return control to FORTRAN. When a single input or output is complete, the FORTRAN program is interrupted and a device handler is executed. If more data values are to be input or output, the device handler initiates the required operations and returns control to FORTRAN.

The X.Y display device requires a further complication. All the display coordinates must be output periodically to refresh the screen phosphor. Thus, output to the display device must be initiated by the clock device handler. When the refresh interval has elapsed, the clock handler initiates output of the coordinates of the first point. Completion of this output causes an interrupt for the display device handler, which outputs the remaining coordinates.

\section{General Service Routines}

The general service routines are designed to perform a wide variety of transformations on visual displays. Displays can be created that appear to move in depth, undergoing both translation and rotation. Other possible displays include both static and dynamic stereoscopic displays, form change during motion, and a large number of transformations of static displays. In keeping with the overall goal of maximizing flexibility, the general service routines are written as a library of subroutines that can be used in innumerable combinations. New routines for special displays can be easily substituted, and subroutine parameters can be set either in the main program or interactively by "housekeeping" programs.

The routines create displays by transforming threedimensional representations of objects in space into two-dimensional representations on the viewing screen. This is accomplished by the combination of four different types of routines: (1) display generators, (2) movement routines, (3) projection routines, and (4) housekeeping routines. Display generator routines create arrays of three-dimensional coordinates that define an object in space. These three-dimensional coordinates can then be transformed by the movement routines to represent movement of the object to a new position in space. All of this is done on the computer's stored threedimensional coordinates of the object. In order to present an image of the object on the viewing screen, we must convert these three-dimensional coordinates into a two-dimensional representation that corresponds 
to coordinates on the viewing screen. As in a painting that uses perspective, we want our two-dimensional image to duplicate the pattern on the retina that is created by a real three-dimensional object. The projection routines calculate such two-dimensional representations for either monocular or binocular viewing. In the first case, a single two-dimensional image is created. For binocular displays, each eye is presented with its own view of the object, thus creating a stereoscopic display.

Continuous motion can be generated by looping through the movement and projection routines, creating a series of static views of a moving object much like the frames of a motion picture. At any point in the cycle, we can change the input parameters for the movement routines, or we can change the shape of the object we are moving. The resulting displays can reproduce motions that are quite complex.

Display generators. To use the general service routines, the laboratory user needs to define coordinates in space that represent an object to be displayed. Often these coordinates are generated by mathematical functions or algorithms programmed by the user. However, two interactive routines in the library permit generation of an object representation from terminal inputs. POINTS creates arrays of points specified by the user at the terminal. This routine is used to create displays that consist of a small number of dots in three-dimensional space. LINES is an interactive routine that connects lines between any points in three-dimensional space. The user interactively specifies a set of points in space and indicates which points are to be connected by lines.

Movement routines. Movement of objects in a threedimensional space is accomplished by two routines, TRANSL and ROTATE. TRANSL increments the $X$, $Y, Z$ coordinates of an object by any desired change in $\mathrm{X}, \mathrm{Y}$, and $\mathrm{Z}$. The routine also keeps track of the cumulative translation that an object has experienced. ROTATE changes the coordinates of the object by rotating the points by specified amounts around an $\mathrm{X}, \mathrm{Y}$, or $\mathrm{Z}$ axis. The input parameters to ROTATE are the amount of rotation about each axis and the coordinates of the center of rotation. ROTATE causes an object to rotate about a point within the object only if the axis of rotation passes through the object. Otherwise, the object revolves around the axis of rotation. To achieve rotation about a point within an object requires the creation of a new coordinate system with an origin always placed at the center of rotation. If we begin with the object's center of rotation placed at the origin of the permanent coordinate system, then TRANSL will keep track of the location of this point. Thus, the object can be made to continuously rotate as it moves in space by using the cumulative totals provided by TRANSL as the center of rotation. Both movement routines can be applied separately to different parts of the total display, thus allowing for different motions by each object in the display.

Projection routines. Up to this point, we have been working with the three-dimensional coordinates of objects moving in space. The retinal pattern that occurs when these objects are observed can be reproduced by an image on a two-dimensional screen. This image is generated by calculating the points on the screen that represent the intersection of the plane of the screen and a set of vectors from the eye to each of the points defining the object. Three projection subroutines are currently part of the library. PROJEC simulates monocular viewing by calculating the intersection of the screen and vectors from the "cyclopean" eye to the points in space that represent our object. DISPAR calculates the intersection of the screen and vectors from both eyes, thus providing a different image for each eye. This program provides both monocular and binocular projective information regarding object distance. For many purposes we may want only the projective information provided by the different position of the two eyes, and not the additional projective change in the vertical dimension that is available in monocular viewing. DISP2 provides such a display. The disparity routines are still under development.

Housekeeping routines. The housekeeping routines serve a variety of purposes. INTMOV is an interactive program that can be used to set all values required by the service routines. The subroutine prompts the user to specify the viewing distance and the amount of translation and rotation. OCOUNT facilitates changes in the shape of an object that has been moved from its original position. The routine keeps track of the cumulative translation and rotation experienced by an object. Thus, the shape of an object can be changed by first manipulating the original coordinates describing the object and moving the object to its current position. By combining instructions to change the shape of an object with OCOUNT and the movement routines, an object can undergo change of shape and motion simultaneously.

A large amount of computation is required to calculate the points for each frame of a moving display. With simple displays these calculations can be performed while the display is in progress. However, with more complex displays movement becomes quite jerky when calculations are done on-line. The alternative is to store the output of the projection routines in disk files and display them after all the frames have been computed. DSKDSP is an interactive program that performs this display function while allowing the user to specify the desired interframe interval. The result is a nice smooth display.

\section{APPLICATIONS}

This laboratory has existed for less than 1 year, and 
some aspects of it are still under development. Still, the experiments that have been conducted or planned for the laboratory demonstrate its versatility. The first experiments were psychophysical studies of temporal order perception. These experiments required precise control of the interval between stimulus onsets or offsets, as well as control of the stimulus intensities. In these experiments subjects judged the order of two visual stimuli or of an auditory stimulus and a visual stimulus. Future experiments will require comparisons between auditory and tactile stimuli or visual and tactile stimuli. Stimulus intensity and the interval between onsets or offsets were manipulated in these experiments.

The software design permits implementation of any psychophysical procedure. We have used the method of constant stimuli, method of limits, and the staircase procedure. The latter two procedures require selection of stimulus parameters based on the previous response. Such response-contingent procedures are not possible in many laboratory systems.

Cognitive research is facilitated by a combination of system capabilities including text presentations, latency measurements, and intensity control. For example, studies of the latencies to discriminate words from nonwords could be conducted. In addition, latencies to complex auditory stimuli can be measured. Thus, discriminations of words and nonwords could be required for spoken stimuli.

The general service routines were developed to permit studies of the information provided by stimulus movement. The retinal transformations that signal changes in shape or depth will be studied. Further studies will examine the effects of stimulus movement in stereoscopic displays. In addition, stereoscopic presentations will be used to discriminate between central and peripheral visual processing. For example, the effects of masking in stereoscopic depth will be investigated.

In summary, this system permits presentation of simple or complex visual, auditory, and tactile stimuli. In the visual modality, stimuli can be generated, moved, and presented stereoscopically. Furthermore, the software contributes to the flexibility of the system by providing service routines that control stimulus presentation and generate displays, but the procedures of an experiment are determined by a program written by the user. 